\title{
Article \\ Simulation-Based Performance Analysis for a Novel AVS/RS Technology with Movable Lifts
}

\author{
Boris Jerman ${ }^{1, *}$, Banu Yetkin Ekren ${ }^{2}\left(\mathbb{D}\right.$, Melis Küçükyaşar ${ }^{2}{ }^{(1)}$ and Tone Lerher ${ }^{3}(\mathbb{C}$ \\ 1 Faculty of Mechanical Engineering, University of Ljubljana, Aškerčeva cesta 6, 1000 Ljubljana, Slovenia \\ 2 Department of Industrial Engineering, Yasar University, No: 37-39, Bornova, Izmir 35100, Turkey; \\ banu.ekren@yasar.edu.tr (B.Y.E.); melis.kucukyasar@gmail.com (M.K.) \\ 3 Faculty of Logistics, University of Maribor, Mariborska c. 7, 3000 Celje, Slovenia; tone.lerher@um.si \\ * Correspondence: boris.jerman@fs.uni-lj.si; Tel.: +386-1-4771-414
}

Citation: Jerman, B.; Ekren, B.Y.;

Küçükyaşar, M.; Lerher, T.

Simulation-Based Performance Analysis for a Novel AVS/RS

Technology with Movable Lifts. Appl. Sci. 2021, 11, 2283. https://doi.org/ 10.3390/app11052283

Academic Editors: Tobias Meisen and Manuel Iori

Received: 14 December 2020

Accepted: 26 February 2021

Published: 4 March 2021

Publisher's Note: MDPI stays neutral with regard to jurisdictional claims in published maps and institutional affiliations.

Copyright: (c) 2021 by the authors. Licensee MDPI, Basel, Switzerland. This article is an open access article distributed under the terms and conditions of the Creative Commons Attribution (CC BY) license (https:// creativecommons.org/licenses/by/ $4.0 /)$.

\begin{abstract}
This paper studies a novel autonomous vehicle-based storage and retrieval system (AVS/RS) design with movable lifts (AVS/RS/ML). In the proposed system, there are aisle-captive lifts that are able to travel along the warehouse aisle to position themselves at the target column location. Those lifts can lift up/down the autonomous vehicles to/from the target storage compartment when they are in standstill. This novel design is proposed as an alternative to existing AVS/RSs to balance the resource utilizations as well as to provide an inexpensive solution with highly utilized autonomous vehicles (i.e., AGVs). As an initial work, for this novel system, two alternative operating designs under different racking configurations are experimented. We compare those two designs by their throughput rate performance metrics under the arrival rate scenarios with highly utilized AGVs (i.e., 95\%). Besides, we experiment with two warehouse capacity scenarios: 900 and 1800 storage compartments. The results show that designs with two separate $\mathrm{I} / \mathrm{O}$ point locations provide a better throughput rate than designs with single I/O point location. Besides, a decreased number of columns in the system improves the system's performance.
\end{abstract}

Keywords: logistics; automated warehousing; AVS/RS; movable lifts; simulation modelling; material handling system

\section{Introduction}

Modern high bay warehouses processing small and light totes usually adopt a shuttlebased storage and retrieval system (SBS/RS), which is an automated warehousing technology with automatic vehicles (i.e., shuttle carriers). Figure 1 shows an SBS/RS. In that system, shuttle vehicles move totes horizontally within a tier aisle. Shuttles are tier-captive vehicles whose main duty is to transport totes from their storage compartments to elevator's lifting tables and vice versa. Lifts (elevator's lifting tables) are located at the periphery of each aisle to transport totes between tiers and input/output (I/O) points.

By a dedicated shuttle vehicle in each tier, SBS/RS provides a high throughput rate. However, on the other hand, works on SBS/RS design show that lifts usually become bottleneck in those systems and shuttle vehicle utilizations are very low [1-3]. To improve that unbalanced condition, a double capacitated lifting system is adopted as shown in Figure 1. However, lifting systems are still mostly the bottleneck resources in those systems.

Different from the described SBS/RS with tier-captive shuttle vehicles, an autonomous vehicle solution with free-moving automated guided vehicle (AGV) that can enter the aisle at the ground floor that can lift itself vertically along each rack column has also been introduced in material handling market [4]. The main disadvantage of that design is the large amount of electricity consumption from their batteries during the lifting operation.

Alternatively, in this paper, we propose a novel autonomous vehicle-based storage and retrieval system (AVS/RS) with movable lifts (MLs) where autonomous vehicles (i.e., AGVs) can travel inside and outside of storage aisles. This system is described in more 
detail in Section 3. It is referred to as AVS/RS with MLs (AVS/RS/ML) throughout the paper.

Note that the purpose of this paper is to study a novel AVS/RS/ML by utilizing a discrete event simulation modelling in order to verify the feasibility of the system and to evaluate the throughput performance for two separate sample designs. Under those designs, we also experiment cases with different number of AGVs as well as different storage rack designs.

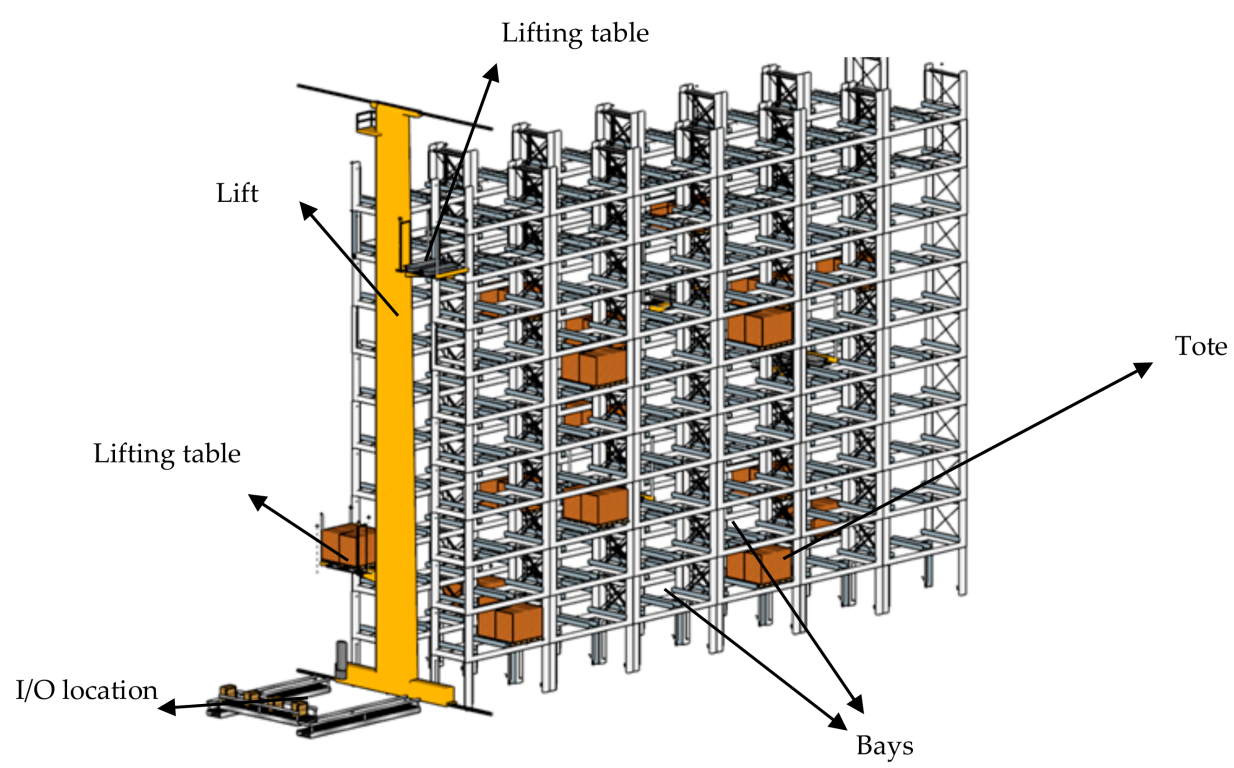

Figure 1. A shuttle-based storage and retrieval system (SBS/RS) from a single aisle view.

Here, the main research questions are:

RQ1-Is there an alternative automated storage and retrieval system design to existing SBS/RS that would be cost efficient and have balanced utilization levels of lifts and autonomous vehicles?

RQ2-By the proposed novel AVS/RS/ML system, by the decreased number of AGVs, since the throughput rate per unit time may decrease, would there be good designs under that proposed novel system helping to increase the throughput performance of the system?

Note that for RQ1, we propose the studied AVS/RS/ML. For RQ2 we study two alternative warehouse designs for that AVS/RS/ML, which are zoning and non-zoning scenarios. Besides, we also experiment different number of AGVs for the non-zoning scenario. To the best of our knowledge, this paper first studies a novel system design developed as an alternative to tier captive SBS/RS. This paper is organised as follows. In Section 2, we provide a literature review about the related works. In Section 3, we describe the proposed novel AVS/RS/ML in detail by its operation rules, main characteristics, and design of MLs and AGVs. In Section 4, we describe the research methodology and the methods used, including the system simulation model assumptions. In Section 5, we provide the results of the experimental work and the discussion. Last, in Section 6, we conclude the work.

\section{Literature Review on AVS/RS}

AVS/RS is first studied by Malmborg [5] by proposing analytical conceptualization tools based on the characteristics of autonomous vehicle systems. He models the system to estimate expected performance metrics as a function of important system attributes such as storage capacity, rack configuration, and fleet size. The models are demonstrated for an exemplary problem and compared with analytical conceptualization tools. In a later study, Malmborg [6] proposed a state equation model to predict the proportion of dual command cycles in autonomous vehicle storage and retrieval systems using interleaving. Although 
the proposed model has some limitations, it is a useful tool for estimating the storage and retrieval cycle times in autonomous vehicle storage and retrieval systems.

Differently, Kuo et al. [7] proposed cycle time models for class-based storage policies in a unit-load automated storage and retrieval (S/R) system. The proposed model is based on a queueing network approach to achieve sufficient accuracy and computational efficiency for system design conceptualisation applications.

By considering non-Poisson arrivals, Zhang et al. [8] investigated approximation strategies for transaction waiting times in Autonomous Vehicle Storage and Retrieval Systems (AVS/RSs). Based on the simulation validation, the proposed strategies can reduce the amount of error in estimated transaction waiting times by about $80 \%$ compared to static approximations.

Different from the above studies, Ekren et al. [9] conducted a simulation-based experimental design for unit-load (UL) automated storage and retrieval systems based on autonomous vehicle technology to identify factors influencing several performance metrics. To benchmark the warehouse solutions, they apply the method of Design of Experiment (DOE). In a later study, Ekren and Heragu [10] study simulation-based design for AVS/RS. They experiment several AVS/RS configurations by simulating their models and present various outputs from the multiple runs. A practitioner may select the best design based on his/her requirements.

By introducing semi-open queueing network with class switching Roy et al. [11] investigated the performance effects of AVS/RS design decisions by using an analytical model. A decomposition-based approach was developed to evaluate system performance of AVS/RS. The numerical studies provide various insights that could be useful in the design of AVS/RS.

Performance comparison of two automated warehousing technologies, AVS/RS and crane-based AS/RS, was presented by Ekren and Heragu [12]. The results show that AVS/RS could provide better performance if the layout is also considered in the warehouse design.

Marchet et al. [13] modelled an SBS/RS by an open queuing network model to estimate the performance of the SBS/RS in terms of lift and shuttle utilisation. For benchmarking the analytical model, they compare the results of the analytical models with the results of the simulation model. The proposed analytical models show good estimates for the performance indicators of SBS/RS. In a later study, Marchet et al. [14] presented the main design trade-offs for SBS/RS by using simulation. They completed their study for several warehouse design scenarios for tier-captive shuttle vehicles.

Different from Marchet et al. [13,14], Lerher [15] proposed an analytical travel time model for calculating single command and dual command cycle times for double-deep Shuttle-Based Storage and Retrieval Systems (SBS/RS). The proposed model takes into account the real operating characteristics of the elevator's lifting table and the shuttle carrier with the condition of rearranging blocking totes to the nearest free storage location during the retrieval process of the shuttle carrier.

Zou et al. [16] proposed a parallel processing policy for autonomous vehicle storage and retrieval systems (SBS/RS), where an arrival transaction can request both the lift (elevator) and the vehicle (shuttle carrier) at the same time. A fork-join queueing network is formulated in which an arrival transaction is divided into a horizontal movement task served by the vehicle and a vertical movement task served by the elevator. The authors use a simulation model to validate the analytical models.

Introducing a multi-elevator tier-captive shuttle-based storage and retrieval system (SBS/RS), Ning et al. [17] developed an efficient simulation model that can be automatically redesigned for different storage rack configurations. The benefit of their model is to test a large number of rack alternatives in order to efficiently determine the optimal solution.

By considering discrete-time open queueing network approach, Epp et al. [18] proposed a method for performance evaluation of autonomous vehicle storage and retrieval 
systems (AVS/RSs) with single aisle tier-captive vehicles. They compare the efficiency of the model with a discrete-event simulation.

In a later study, Lerher [19] investigated aisle-changing shuttle carriers in AVS/RSs. The author proposes analytical travel time models for aisle-changing shuttle carriers, which can travel in both horizontal and cross-aisle directions. The expressions for the travel times of single and double commands are determined under the assumption of uniformly distributed storage locations and the theory of probability. A simulation model is developed to benchmark the proposed analytical models.

Very recent papers on SBS/RS were completed by Ekren [20]. Ekren et al. [1,2,21] proposed a tool for calculating critical performance measures of various shuttle-based storage and retrieval system (SBS/RS) designs. The proposed methodology can also be used for any system with a linear, discrete travel pattern with stop points of equal distance. Utilizing the proposed tool, the performance of SBS/RS systems can be immediately evaluated by changing the input design parameters of SBS/RS. In a later study, Ekren and Akpunar [22] presented a queuing network-based tool estimating several performance metrics from a pre-defined SBS/RS design. The developed tool can be downloaded from a website for free. Besides those, to show the effectiveness of class-based storage policy, Ekren et al. [3] studied SBS/RS warehouse under several storage policies.

Recently, Küçükyaşar et al. [23,24] investigated two SBS/RS configurations (e.g., tiercaptive shuttles with tier-to-tier shuttles). The authors proposed an alternative SBS/RS design with tier-to-tier shuttles, where the shuttles can travel between tiers by using a separate lifting mechanism dedicated to them. The proposed results suggest that a newly proposed tier-to-tier SBS/RS design can provide a better configuration in terms of total investment cost and throughput rate.

The existing works mostly focus on design and modelling approaches for AVS/RS and SBS/RS. For instance, simulation modelling approach is one of the most powerful tools utilized for modelling of such complex systems. By that approach, several different designs could be tried for systems that could be hard to model analytically. Compared to the existing literature on AVS/RS works [5,8,10-12,18,19], the proposed research considers a novel AVS/RS technology design with MLs. These aisle-captive lifts are, only while not loaded, able to travel along the warehouse aisle to position themselves at the target column location. Further, they can, only when they are at the standstill, lift up the systems autonomous vehicles to the target tier and lower them down to the aisle floor. To the best of our knowledge, this is the first study presenting that novel AVS/RS technology design with movable lifts, ML, and analyzing the efficiency of the proposed system with simulation under different operational aspects. For the modelling approach, we utilize simulation modelling approach due to its power in modelling such complex systems.

\section{The Novel AVS/RS/ML System}

\subsection{Description of the System}

In this paper, we propose a novel autonomous vehicle-based storage and retrieval system design with movable lifts (AVS/RS/ML) that could be utilized in high bay mini-load warehouses (see Figure 2). The proposed system is employed for storage (i.e., transport of totes with items from points of delivery to storage compartments) and retrieval operations (i.e., transport of totes with items from storage compartments to order-picking locations or directly to the production assembly line). Within this system, the autonomous vehicles (i.e., AGVs) can travel inside and outside of storage racks. AGVs are utilized for processing of storage and retrieval operations as well as for transport and distribution of totes throughout the production plant.

According to that new system proposal, AGVs can make horizontal travel within an aisle floor and can be lifted vertically to reach upper levels of the storage racks by the help of MLs located in each aisle. To perform lifting process, AGVs attach themselves to the ML's lifting chains. For that, AGVs would be equipped with appropriate telescopic attachment (Figures 3-5) for automated manipulation with totes, retractable grippers for 
clamping on the lifting chains, and with stoppers for its anchoring on the rack at the target location (Figure 3).

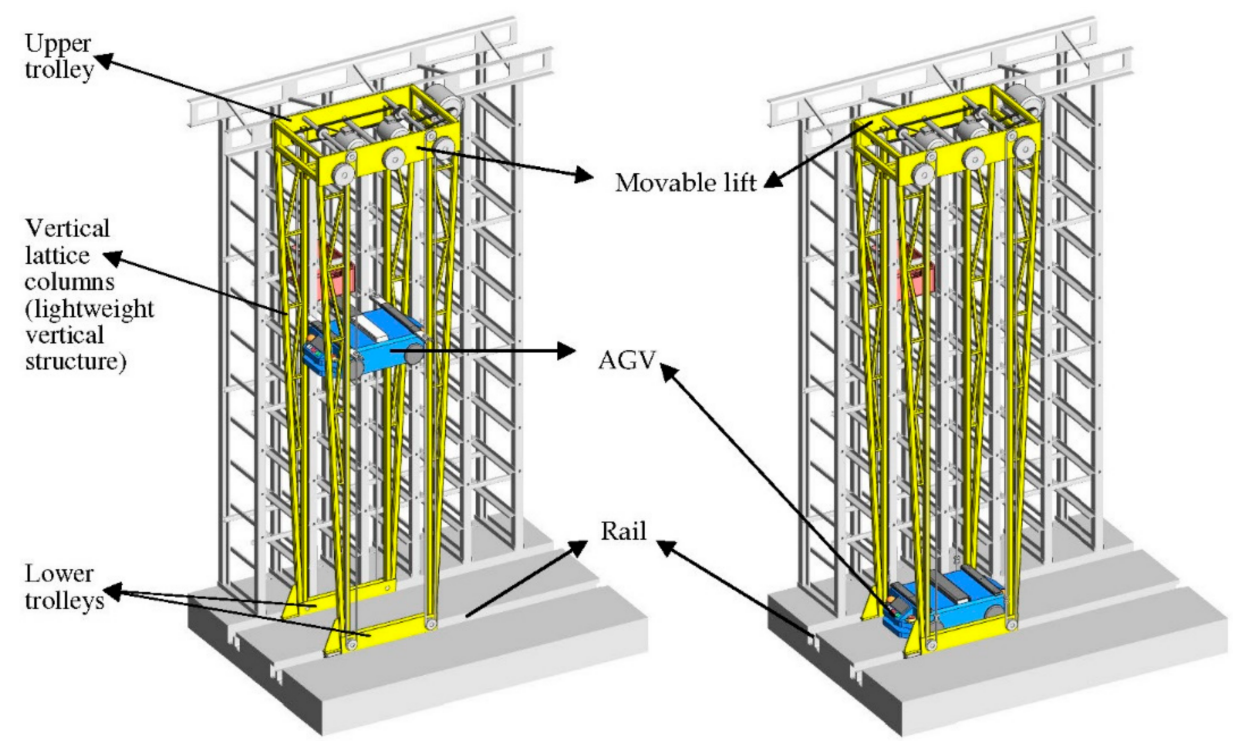

Figure 2. The proposed autonomous vehicle-based storage and retrieval system design with movable lifts (AVS/RS/ML).

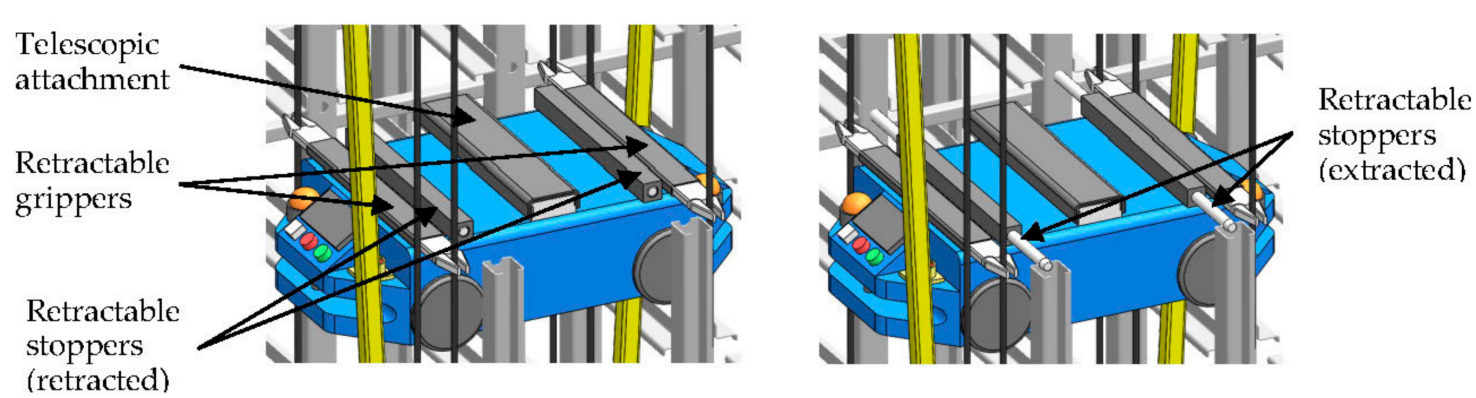

Figure 3. The AGV clamped on the ML's lifting chains by grippers (lattice columns are partially hidden).

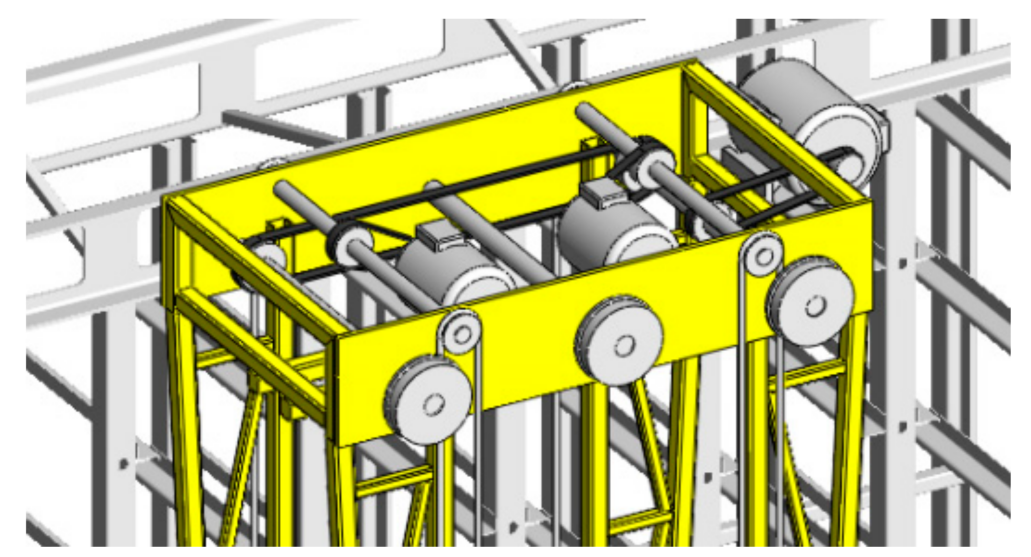

Figure 4. Upper trolley with driving, braking, and lifting mechanisms.

In each warehouse aisle, there can be one or multiple aisle-captive MLs. By assigning different number of MLs to each aisle, the utilizations of material handling resources in the system can be balanced. When more than one ML is considered in an aisle (on the same rail), proper collision prevention algorithms would need to be considered. 
In the proposed AVS/RS/ML, there is no need to use expensive stacker cranes or complicated vertical lifting mechanisms together with aisle-captive shuttle vehicles. Those are relevant advantages compared to the existing autonomous vehicles or stacker cranesbased storage and retrieval systems. When we compare the introduced novel AVS/RS/ML with the recently developed system with AGVs [4], it is also a great advantage that AGVs lifted by MLs would not need to be equipped with their own lifting mechanisms and they do not consume electricity from their batteries during that lifting process. When AGVs are attached on the MLs, AGV batteries can also be charged by conductor bars and collectors attached along ML's lattice columns and on AGVs, respectively.

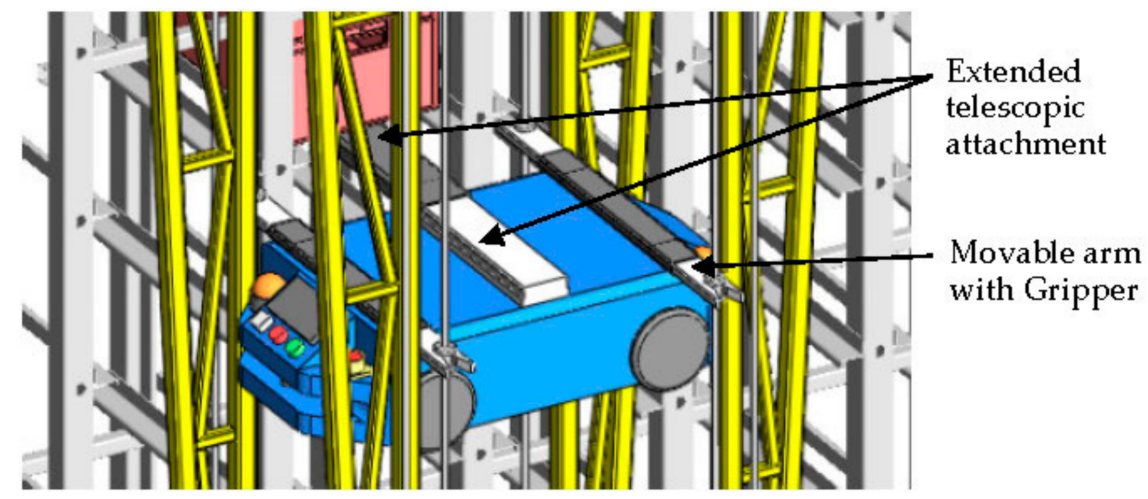

Figure 5. Lifted AGV at the target level, with telescopic attachment in extended position.

\subsection{Description of the Operation of the System}

The main motivation of the newly proposed system is to apply technically simple and economically affordable portable lifting devices (i.e., MLs) together with higher number of vehicles (i.e., AGVs) to balance utilization of those both devices in the system and to provide higher throughput with less expenses. Here, AGVs can travel inside the warehouse aisles as well as on the factory floor outside of the proposed AVS/RS/ML. When AGV receives a storage transaction request, first it picks up the tote at the factory floor or at the I/O point and travels to the storage address aisle. After it enters the aisle, it travels to the target column. If the target storage compartment is at an upper level, an available ML is directed to move to the regarding column simultaneously with the AGV. When both the ML and the AGV arrive at the target column location, the AGV clamps itself on the ML's lifting chains and it is lifted to the target storage compartment by the ML. After the storage process is completed, the ML lifts down the AGV to the ground level. After the lift reaches at the ground floor, the empty AGV releases itself from the ML's lifting chains, it leaves the warehouse aisle, and waits for further instructions. The retrieval transaction is performed in an identical manner. However, the difference is that the AGV is empty when it enters the aisle. However, it leaves the aisle with a tote.

The horizontal movement of MLs is relatively slow. To prevent excess waiting times of AGVs, as well as for further busting of the throughput performance of the AVS/RS/ML, ability of the ML to lift up two AGVs at the same time can be implemented. For that, AGVs should be equipped with retractable stoppers (see Figure 3) by which the AGV can be anchored to the rack construction. For successful implementation of this solution, the system logic must ensure that the first AGV attached should manipulate the tote in higher storage compartment than the second one. Hence, AGVs do not collide during their lifting process. Appropriate anti-collision algorithms must also be considered while AGVs' descending. In the present research, these features have not yet been included however proposed as future works. Here, simpler anti-collision principles were implemented, as explained in Section 4. 


\subsection{Description of the Movable Lift $(M L)$}

The ML (Figure 2) is the main innovation of the proposed novel design of AVS/RS. It consists of upper trolley (Figures 2 and 4), the lightweight vertical structure (Figure 2) and two lightweight lower beam trolleys (Figure 2). The design of the upper trolley is similar to the design of simple overhead crane's trolley with driving, braking, and lifting mechanisms, where instead of a large and expensive wire rope drum, only four sprocket wheels are implemented. The driving mechanism of the upper trolley has four wheels, running on the lower flanges of the U-shaped rails and two wheels running on the upper flanges providing exact and stable position of the trolley during ML's operations in standstill, during decelerations and accelerations of horizontal movements, and during constant velocity travel. There is a lightweight vertical structure which mainly consists of four vertical lattice columns. The top ends of these four columns are connected to the upper trolley and the bottom ends are connected to two lower beam trolleys. Lower beam trolleys are designed as horizontal beams equipped with guiding side wheels, brakes, and four return sprocket wheels. The guiding wheels and the brakes are acting on the lower rail, which is built-in into the aisle floor. Four lifting chains are tensioned over sprocket wheels on the upper trolley and return sprocket wheels on the lower beam trolleys.

The ML is capable of performing horizontal travel to reach the target column location when it is empty. When it is in standstill, it is capable of lifting an AGV. The main and very significant difference between the ML and the stacker crane therefore is that ML is able to lift payload only when it is in standstill. In addition, the horizontal velocity of ML is smaller than that of the stacker crane. According to this, it can be assumed that the ML can be produced cheaper compared to the lifting mechanism in SBS/RS and mini-load stacker crane of an automated storage and retrieval system.

The MLs are structured slender and lightweight and have relatively small accelerations and decelerations of horizontal travel velocity. Further, they are equipped with brakes on the upper and lower trolleys, enabling precise positioning of the ML and more rigid construction during standstill. More rigid construction is provided by also designing vertical columns as lattice girders. Keeping all this in mind, we assume that oscillations of the ML's columns fade out fast enough. To prevent excessive horizontal oscillations of vertical lifting chains, simple dumpers, connected to the ML's lattice columns can be employed.

To test its performance, this proposed novel system's working principle is simulated under computer environment. Different operating policies are considered to observe how performance metrics of the system are affected.

\section{Analyses of the Novel AVS/RS/ML}

In this section, simulation model along with the considered assumptions and limitations for the proposed AVS/RS/ML are explained. The simulation model of the system is developed by using the ARENA 16.00 commercial simulation software.

\subsection{Simulation Model of the Proposed AVS/RS/ML}

In the simulation model, we assume that there are two MLs per aisle. For the easiest way of collision prevention of MLs, in one design, a warehouse aisle is divided into two separate zones and each of the MLs operates in a dedicated zone. Furthermore, in another design, we assume that there are more than a single AGV in the system. Remember that there are two types of transaction requests arriving at the system: Storage and retrieval. The processes completed for those requests are summarized below.

Storage requests:

- An available AGV, waiting at the I/O point, is selected randomly by the arriving storage transactions.

- While transaction is charged at the selected AGV, an appropriate ML is also activated and both travel to the target column location simultaneously. As a note, if the storage compartment is at the ground level, ML is not utilized for that transaction. 
- $\quad$ AGV with the tote is clamped on the lifting mechanism and it is lifted to the target tier of the storage rack. Note that once the AGV reaches the target tier, it is at the position of the required storage compartment at the same time.

- $\quad$ AGV drops off the tote at the storage compartment.

- $\mathrm{AGV}$ is moved downwards at the ground level and the ML is released.

- AGV travels to the output point to take another waiting transaction. If there is no waiting tote, the AGV waits idle in the system.

Retrieval requests:

- An available AGV waiting at the I/O point is selected randomly by an arriving retrieval transaction.

- While the selected AGV travels to the requested column of the target storage compartment, it also requests the ML; if it is seized, then both travel to the regarding column simultaneously.

- $\quad \mathrm{AGV}$ is clamped on the lifting mechanism of the ML, and it is lifted up to the target tier of the storage rack. Note that once the AGV reaches the target tier, it is at the position of the required storage compartment at the same time.

- $\quad$ AGV picks up the tote at the storage compartment.

- $\quad$ AGV is lifted down at the ground level and the ML is released.

AGV travels to the output point and drops off the tote.

The details of the operating rules considered in the simulation are shown in a flow chart in Figure 6.

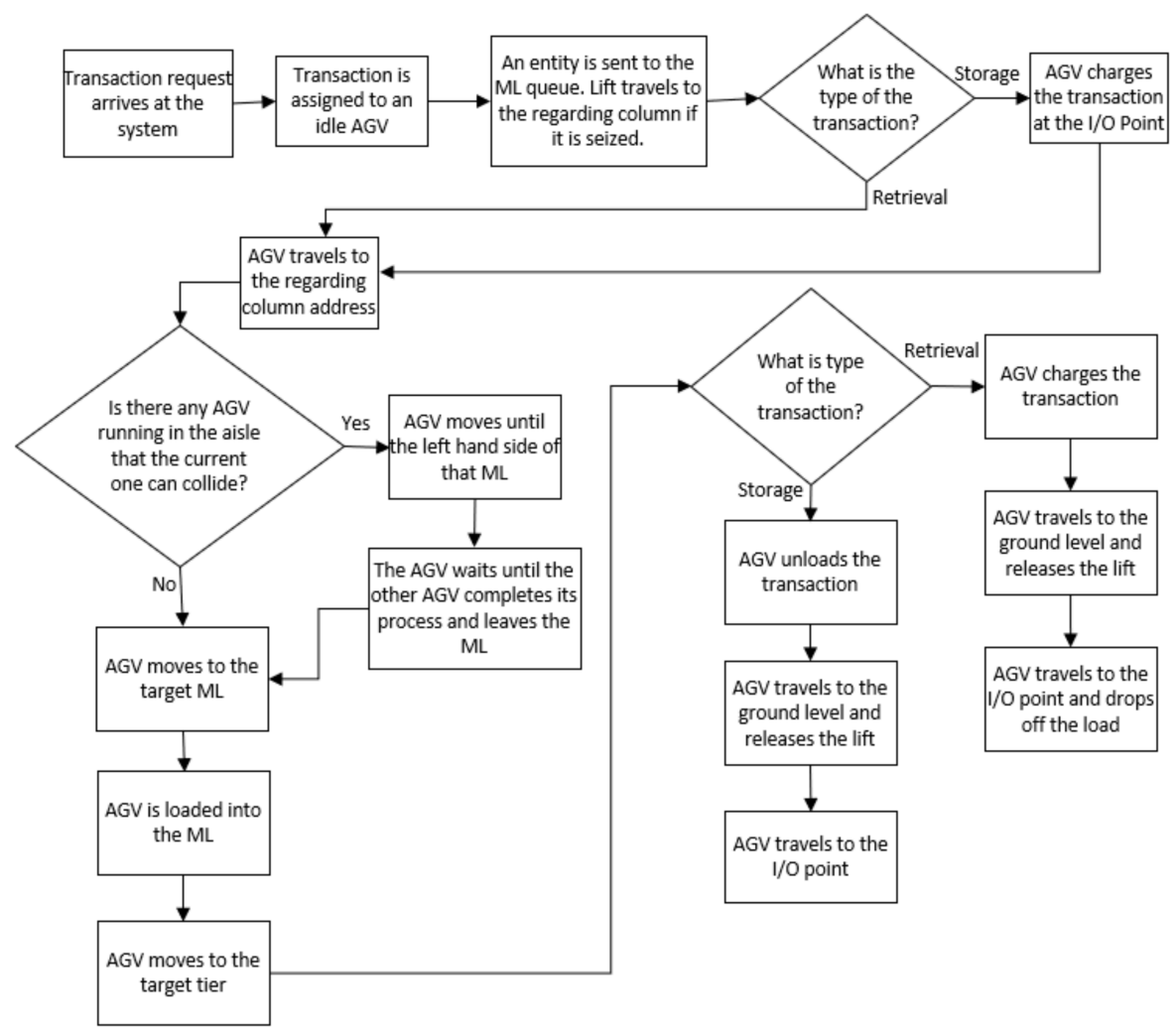

Figure 6. Flow chart of storage and retrieval transaction requests.

By simulating the system, we aim to compare two alternative operating designs for the described system. In the first design (Figure 7a), we assume a single I/O point that is located at periphery of enter point of each aisle. In the second design (Figure $7 \mathrm{~b}$ ), we assume that there are two separated I/O points, located at both enter points of a divided zone. It is clear that in both designs, there is an extra space at the middle of each aisle, needed for prevention of collision of two MLs (e.g., note that the width of MLs get larger at 
upper levels; see Figure 2). Note that, in the Figure 7a design, we also allow AGVs to leave the aisle at the middle point to travel to the I/O point. In this design, we also consider one-way travel pattern for AGVs so that, while they enter the warehouse aisle from the I/O point side, they leave it either form the middle or from the other end side according to their last completion location.

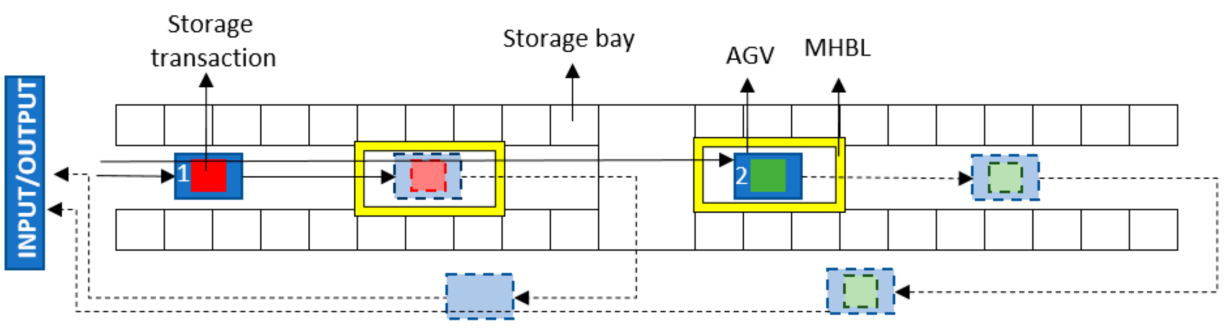

a) System design with single I/O Point

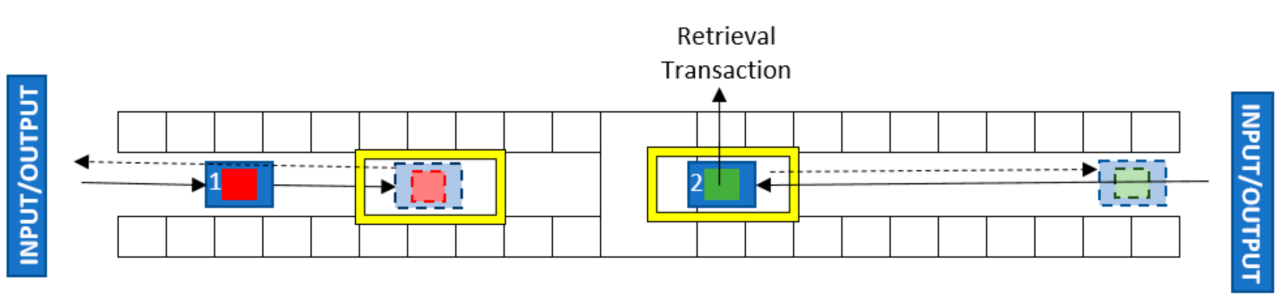

b) System design with two I/O Points

Figure 7. Top views of system designs with single and two input/output (I/O) points.

The design with single $\mathrm{I} / \mathrm{O}$ location is experimented with two and three AGVs. For simple prevention of collision of the AGVs, it is assumed that an AGV cannot pass through another AGV that is in front of it. It stops and waits until that AGV completes its process even if that $A G V$ is in the lifting mechanism.

The design with two I/O locations is modelled by considering two AGVs each of which is dedicated to a divided zone. Hence, there is no possibility of collision of two AGVs. In this design, after AGVs complete their process they do not move to the I/O position to wait for further deployment. Instead, they stay at the ground location where they completed their last task.

For each of different physical configurations based on I/O locations, we also considered different rack configurations with different number of tiers and columns scenarios.

\subsection{Details of the Simulation Model}

In this section, we detail the simulation models of the proposed designs. Figures 8 and 9 show the screenshots from the animated model of a warehouse aisle with 30 columns and 15 tiers of the storage rack.

The AVS/RS/ML system design with single I/O point includes two different control policies for collision prevention for the cases of two or three AGVs in this system. If there are two AGVs in the warehouse aisle, the newly entering AGV first checks the location of $\mathrm{AGV}$ in the aisle. If an AGV is not standing on the way to the location of the prescribed storage column of that newly entering AGV, then that AGV enters the aisle and travels to the prescribed storage column to process the assigned transaction. The third AGV always waits at the I/O point, if there are two AGVs already in the aisle. There can be three AGVs in the warehouse aisle at the same time only in the case that one of them is traveling towards the I/O point at the ground level. Doing so, traffic collision control is realized in the simplest way. 


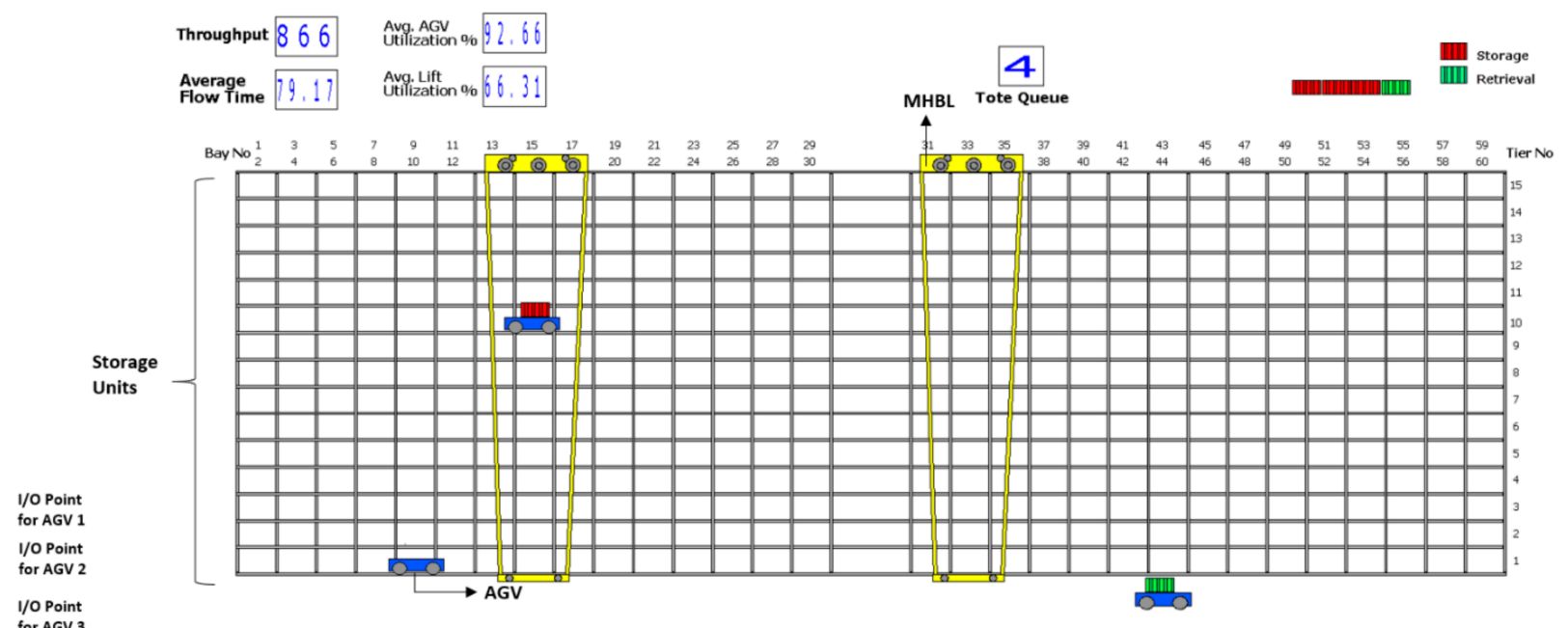

Figure 8. A screenshot from the animation of the simulation model with single I/O point.

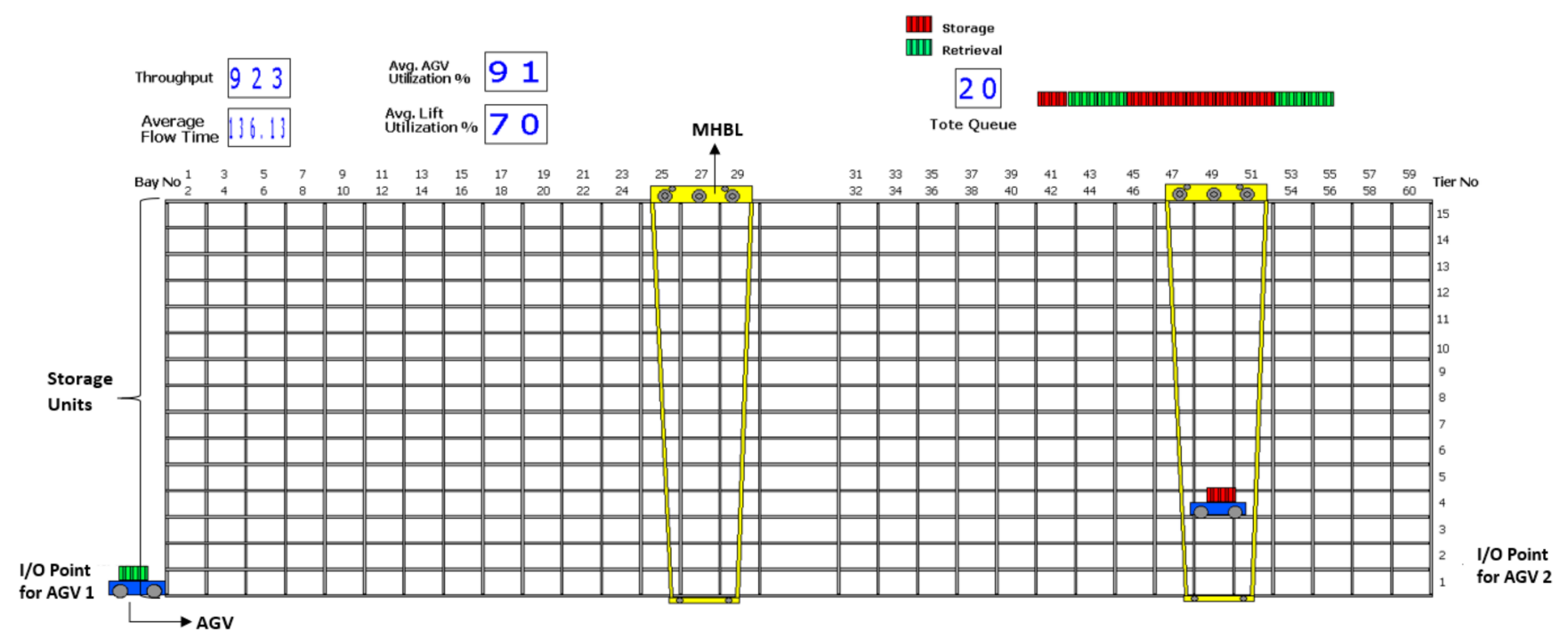

Figure 9. A screenshot from the animation of the simulation model with two I/O Points.

The other considered assumptions are as following:

- Arrivals of demand follow Poisson process with equal mean of storage and retrieval transactions.

- Random storage assignment policy is considered for arriving transactions.

- If the storage or retrieval sequence of the processed transaction is at the first tier of the storage rack, then ML will not be utilized.

- The maximum horizontal velocities of AGVs and MLs are $3 \mathrm{~m} / \mathrm{s}$ and $1.5 \mathrm{~m} / \mathrm{s}$, respectively. The maximum vertical velocity of the AGV, when it is loaded on the ML, is $2 \mathrm{~m} / \mathrm{s}$.

- Accelerations and decelerations of AGVs and MLs are considered as $2 \mathrm{~m} / \mathrm{s}^{2}$ and $1 \mathrm{~m} / \mathrm{s}^{2}$, respectively.

- The distance between two adjacent bays is equal to $0.5 \mathrm{~m}$. The distance between two adjacent tiers is assumed to be $0.35 \mathrm{~m}$.

- $\quad$ Pick-up and drop-off times for totes equal to $t_{\mathrm{P}}=3 \mathrm{~s}$ and $t_{\mathrm{S}}=3 \mathrm{~s}$.

- Gripping and ungripping times of the AGV equal $t_{\mathrm{g}}=1 \mathrm{~s}$ and $t_{\mathrm{ug}}=1 \mathrm{~s}$. 


\subsection{Design Scenarios and Performance Metrics of the Systems}

In this research, we propose a novel AVS/RS/ML—an AVS/RS with movable lifts (MLs). To investigate the system properly, some critical performance metrics are observed from the system. Performance metrics are defined to be throughput rate per month, average flow time of a transaction, average operating time of an AGV per transaction, and average utilization of devices (e.g., MLs and AGVs). Flow time of a transaction is defined to be the time from arrival of a transaction (storage/retrieval) at the system until completion of its process. Hence, that flow time metric also includes waiting time of transactions in queues. Operating time of an AGV is defined to be the time between when an AGV is seized by a transaction until it returns to its dwell point. For instance, when there is no waiting time for a transaction in the system, the flow time performance metric would always be smaller than the AGV's operating time. This is because AGV's travel time also includes travel time to its dwell point.

Average utilization of an AGV and an ML are obtained by total busy time divided by total simulation time (i.e., one month). Table 1 shows the notations of the performance metrics and their considered units.

Table 1. Notation and definition of performance metrics.

\begin{tabular}{ccc}
\hline Notation & Definition & Unit \\
\hline$t_{f}$ & Average flow time per & transaction \\
$t_{o}$ & Average operating time of an & seconds \\
& AGV per transaction & seconds \\
$U t i l_{A G V}$ & Average utilizations of an & $\%$ \\
$U t i l_{M L}$ & AGV & $\%$ \\
$\lambda$ & Average utilization of an ML & Number of \\
& Average throughput rate & transactions $/$ month \\
\hline
\end{tabular}

These performance metrics are observed in all described system designs of AVS/RS/ML subject to different physical configurations. All these scenarios are detailed in the following sections. The physical configuration parameters are considered to be the number of tiers $(T)$, number of storage columns (SC), capacity of warehouse $(W)$, and the number of AGVs $(N)$; see Table 2.

Table 2. Notations and definitions of parameters.

\begin{tabular}{cc}
\hline Notation & Definition \\
\hline$T$ & Number of tiers in an aisle \\
$S C$ & Number of storage columns in an aisle \\
$W$ & Warehouse capacity of an aisle \\
$N$ & Number of AGVs in an aisle \\
\hline
\end{tabular}

All experimented scenarios along with their data considered are given in Table 3. In the first design scenario, as mentioned, two designs based on I/O point locations are denoted as "system design". We also denote two warehouse capacity scenarios $(W)$ as small and large. In small and larger capacity cases, we consider that there are 900 and 1800 storage locations, respectively. The number of tiers $(T)$ and the number of storage columns (SC) are predefined by dividing $W$ by $2 . T$ because there are racks on both sides of each aisle. There can be a larger number of AGVs in the AVS/RS/ML system. However, we model two and three AGVs in the single I/O point designs and two AGVs in the two I/O points design. Finally, we have 12 scenarios to analyse as given in Table 3. The simulated experimental results are also given in Table 3. 
Table 3. Results of the scenarios.

\begin{tabular}{|c|c|c|c|c|c|c|c|c|c|c|}
\hline $\begin{array}{l}\text { Scenario } \\
\text { No }\end{array}$ & $\begin{array}{l}\text { System } \\
\text { Design }\end{array}$ & $W$ & $T$ & $S C$ & $N$ & $U t i l_{A G V}$ & $\mathrm{Util}_{M L}$ & $t_{f}(\mathbf{s})$ & $t_{o}(\mathrm{~s})$ & $\begin{array}{c}\lambda \text { (Number of } \\
\text { Transaction/Month) }\end{array}$ \\
\hline 1 & & 900 & 10 & 45 & 2 & $95 \% \pm 0.15 \%$ & $70 \% \pm 0.18 \%$ & $279.41 \pm 28.5$ & $16.313 \pm 0.007$ & $301,782 \pm 425$ \\
\hline 2 & Two I/O & 900 & 15 & 30 & 2 & $95 \% \pm 0.12 \%$ & $74 \% \pm 0.12 \%$ & $300.11 \pm 8.61$ & $16.010 \pm 0.004$ & $309,000 \pm 328$ \\
\hline 3 & points & 1800 & 20 & 45 & 2 & $95 \% \pm 0.18 \%$ & $75 \% \pm 0.17 \%$ & $317.80 \pm 34.68$ & $18.200 \pm 0.008$ & $270,382 \pm 441$ \\
\hline 4 & & 1800 & 25 & 36 & 2 & $95 \% \pm 0.15 \%$ & $76 \% \pm 0.14 \%$ & $246.17 \pm 30.65$ & $18.330 \pm 0.007$ & $267,630 \pm 466$ \\
\hline 5 & & 900 & 10 & 45 & 2 & $95 \% \pm 0.10 \%$ & $45 \% \pm 0.02 \%$ & $170.88 \pm 12.47$ & $27.661 \pm 0.008$ & $178,814 \pm 191$ \\
\hline 6 & & 900 & 15 & 30 & 2 & $95 \% \pm 0.07 \%$ & $49 \% \pm 0.06 \%$ & $140.12 \pm 6.21$ & $25.176 \pm 0.017$ & $195,162 \pm 233$ \\
\hline 7 & & 900 & 10 & 45 & $\overline{3}$ & $95 \% \pm 0.26 \%$ & $61 \% \pm 0.17 \%$ & $132.38 \pm 3.72$ & $30.143 \pm 0.028$ & $244,882 \pm 599$ \\
\hline 8 & Single I/O & 900 & 15 & 30 & 3 & $95 \% \pm 0.27 \%$ & $66 \% \pm 0.15 \%$ & $124.49 \pm 3.63$ & $28.586 \pm 0.029$ & $257,146 \pm 551$ \\
\hline 9 & Point & 1800 & 20 & 45 & 2 & $95 \% \pm 0.11 \%$ & $48 \% \pm 0.06 \%$ & $185.51 \pm 12.04$ & $30.109 \pm 0.016$ & $164,148 \pm 206$ \\
\hline 10 & & 1800 & 25 & 36 & 2 & $95 \% \pm 0.11 \%$ & $51 \% \pm 0.03 \%$ & $186.46 \pm 11.83$ & $29.020 \pm 0.021$ & $170,590 \pm 225$ \\
\hline 11 & & 1800 & 20 & 45 & 3 & $95 \% \pm 0.20 \%$ & $66 \% \pm 0.13 \%$ & $160.41 \pm 10.75$ & $33.679 \pm 0.020$ & $219,952 \pm 350$ \\
\hline 12 & & 1800 & 25 & 36 & 3 & $95 \% \pm 0.23 \%$ & $68 \% \pm 0.14 \%$ & $149.77 \pm 8.84$ & $33.444 \pm 0.033$ & $219,952 \pm 350$ \\
\hline
\end{tabular}

Note: the warehouse volume $W=900$ totes and $W=1800$ totes correspond to warehouse volume in a single aisle AVS/RS/ML system design.

\section{Results and Discussion}

In this section, we provide the results of the simulations for the considered scenarios. We perform a "steady-state" analysis, by defining the "warm-up" periods in the designs. We observe that the average flow time per transaction reaches steady after 15 days run time by using the output analyser statistics in the software. We aim to complete our analyses for one month simulation runs. Hence, with the warm-up period, our replication runs are set for 45 days. To analyse the systems under highly utilized conditions, note that the models run for arrival rates so that we obtain larger than or equal to $95 \%$ utilization for the bottleneck devices (i.e., AGVs). Namely, the arrival rate is adjusted so that we obtain at least $95 \%$ average utilization for the AGVs. Table 3 shows the numerical results of the scenarios at $95 \%$ confidence intervals. In Arena software, output variability is reported via the half width, which is half of the 95th percentile confidence interval for the related performance metric.

From the results, we can draw a general conclusion that the warehouse design with novel AVS/RS/ML can produce reasonable throughput rates even there are few numbers of vehicles in the system designs. In both AVS/RS/ML system designs (i.e., with single $\mathrm{I} / \mathrm{O}$ and two I/O points), it is observed that the bottleneck is always the AGVs (i.e., having higher utilization values than MLs). Since there are two MLs and two or three AGVs in the system, probably because the total travel distance of AGVs is usually longer than that of the MLs, they become bottleneck. The fact that the AGVs are bottleneck can also be revealed by comparison of the scenarios of 6 and 8 where it can be seen that when the third AGV is included in the system the throughput increases for more than $30 \%$.

The number of transactions that could be processed per month $(\lambda)$ is always higher in two I/O points scenarios than in single I/O point scenarios regardless of the fact that the configuration of the racks and the number of MLs is the same. This is because the average operating time of an AGV per transaction $\left(t_{o}\right)$ in two I/O points system design is higher than the other system design. Furthermore, note that the dwell point policy of both designs is different. In the single I/O design, AGV always returns to the I/O point after it discharges the transaction at the storage compartment whereas in the two I/O points design the AGV can wait inside the aisle. Therefore, in the single I/O design an AGV spends more time in travel than the other system design.

Two I/O point design has an advantage in terms of throughput rate. However, it does not produce better average flow time per transaction $\left(t_{f}\right)$ performance metric than the single I/O point system design. This is because under that design, we increase the mean arrival rate at the system, causing increased waiting time of transactions in queues-a transaction spends almost $95 \%$ of its $t_{f}$ for waiting in the queue of AGV.

The other inferences can be summarized as follows:

- $\quad$ The studied novel AVS/RS/ML system provides reasonably good throughput rates under highly utilized levels of AGVs although the system works under a restrictive anti-collision algorithm. Once the operating policies and scheduling of transactions will be optimized, those values will be improved significantly. 
- It can be observed from the results that even under highly utilization levels of AGVs, the two devices' utilizations are not that far away from each other as in the traditional SBS/RS. Furthermore, the novel system's design is flexible in the number of MLs and AGVs as well as in their velocities for further balancing of the device utilizations.

- $\quad$ The smaller warehouse capacity ( $W=900$ totes) provides more throughput per month and less operating time of an AGV per transaction compared to the larger one ( $W=1800$ totes). This is mainly because of the decreased travel time of AGVs and MLs.

- For both warehouse capacities, the system design with two I/O points works better in terms of $t_{0}$ and $\lambda$. The two I/O points design scenarios provide roughly $62 \%$ better performance in terms of throughput rate performance metric than the single I/O point scenarios.

- Increased number of AGVs provides better results (i.e., at least $25 \%$ improvement in terms of $\lambda$ and $20 \%$ in terms of $t_{f}$ ) in the single I/O point design.

- In all scenarios, the utilization levels are $95 \%$ for AGVs, and significantly less for lifts ( $48 \%$ to $76 \%)$.

- The conclusions above confirm our opinion that when more efficient routing congestion algorithms and a higher number of AGVs will be included in the system, a much better performance from the novel AVS/RS/ML system can be demonstrated.

\section{Conclusions}

In this paper, a novel AVS/RS with movable lifts (AVS/RS/ML) is proposed as an alternative design to existing AVS/RSs. In the proposed design, it is aimed to provide an inexpensive AVS/RS solution with highly utilized AGVs, capable of warehouse operations and can travel out-of-warehouse as transportation service. Two alternative designs based on I/O locations are experimented by simulating the systems under different racking configurations and with different number of AGVs in the model. The results are compared mainly based on their throughput rate outputs under high utilization levels of AGVs (i.e., 95\%). Two designs are experimented under different warehouse aisle volumes: 900 and 1800 totes. Besides, for each capacity, different number of tiers and columns are also experimented.

From the simulation results, it is observed that in the proposed AVS/RS/ML, average utilization of AGVs is much higher than average utilization of the shuttle vehicles in the traditional SBS/RS. Hence, the performance of the proposed system should be explored more, by comparing its performance throughput rate with the other existing system designs. Besides, integrating smarter operating as well as routing congestion algorithms by comparing with the current simulated ones may also be explored to check whether or not it assures further significant improvements in the system performance.

As a future work, more AGVs can be allowed to travel in an aisle by integrating optimal collision prevention algorithms. Increased number of AGVs can further be balanced with additional MLs. Besides, different racking designs as well as velocity profiles can also be experimented as future works. Other aspects that would be worth focusing on as future works are operating and initial investment costs, safety costs, energy expenses, and space utilizations of that novel system.

Author Contributions: Conceptualization, B.J. and T.L.; methodology, B.Y.E. and M.K.; software, M.K.; validation, B.Y.E. and M.K.; formal analysis, B.J., B.Y.E., M.K., and T.L.; investigation, B.J.; resources, B.J., B.Y.E., M.K., and T.L.; data curation, B.J., B.Y.E., M.K., and T.L.; writing-original draft preparation, B.J., B.Y.E., M.K., and T.L.; writing-review and editing, B.J., B.Y.E., M.K., and T.L.; visualization, B.J. and M.K.; supervision, B.J., B.Y.E., and T.L.; project administration, B.J. and T.L.; funding acquisition, B.J. and T.L. All authors have read and agreed to the published version of the manuscript.

Funding: This research work was supported by the Slovenian Research Agency (ARRS) in the framework of the applied research project entitled: "Warehousing 4.0-Integration model of robotics and warehouse order-picking systems"; Grant number: L5-2626. 
Informed Consent Statement: Not applicable.

Conflicts of Interest: The authors declare no conflict of interest.

\section{References}

1. Ekren, B.Y. Graph-based solution for performance evaluation of shuttle-based storage and retrieval system. Int. J. Prod. Res. 2017, 55, 6516-6526. [CrossRef]

2. Ekren, B.Y. A Simulation-based experimental design for SBS/RS warehouse design by considering energy related performance metrics. Simul. Model. Pract. Theory 2020. [CrossRef]

3. Ekren, B.Y.; Sari, Z.; Lerher, T. Warehouse design under class-based storage policy of shuttle-based storage and retrieval system. IFAC-PapersOnLine 2015, 48, 1152-1154. [CrossRef]

4. Exotec. Available online: https://www.exotec.com/ (accessed on 10 November 2020).

5. Malmborg, C.J. Conceptualizing tools for autonomous vehicle storage and retrieval systems. Int. J. Prod. Res. 2002, 40, 1807-1822. [CrossRef]

6. Malmborg, C.J. Interleaving rule dynamics in autonomous vehicle storage and retrieval systems. Int. J. Prod. Res. 2003, 41, 1057-1069. [CrossRef]

7. Kuo, P.H.; Krishnamurthy, A.; Malmborg, C.J. Performance modelling of autonomous vehicle storage and retrieval systems using class-based storage policies. Int. J. Comput. Appl. Technol. 2008, 31, 238-248. [CrossRef]

8. Zhang, L.; Krishnamurthy, A.; Malmborg, C.J.; Heragu, S.S. Variance-based approximations of transaction waiting times in autonomous vehicle storage and retrieval systems. Eur. J. Ind. Eng. 2009, 3, 146-168. [CrossRef]

9. Ekren, B.Y.; Heragu, S.S.; Krishnamurthy, A.; Malmborg, C.J. Simulation based experimental design to identify factors affecting performance of AVS/RS. Comput. Ind. Eng. 2010, 58, 175-185. [CrossRef]

10. Ekren, B.Y.; Heragu, S.S. Simulation-based performance analysis of autonomous vehicle storage and retrieval system. Simul. Model. Pract. Theory 2011, 19, 1640-1650. [CrossRef]

11. Roy, D.; Heragu, S.S.; Krishnamurthy, A.; Malmborg, C.J. Performance analysis and design trade-offs in warehouses with autonomous vehicle technology. IIE Trans. 2012, 44, 1045-1060. [CrossRef]

12. Ekren, B.Y.; Heragu, S.S. Performance comparison of two material handling systems: AVS/RS and CBAS/RS. Int. J. Prod. Res. 2012, 50, 4061-4074. [CrossRef]

13. Marchet, G.; Melacini, M.; Perotti, S.; Tappia, E. Analytical model to estimate performances of autonomous vehicle storage and retrieval systems for product totes. Int. J. Prod. Res. 2012, 51, 4365-4387. [CrossRef]

14. Marchet, G.; Melacini, M.; Perotti, S.; Tappia, E. Development of a framework for the design of autonomous vehicle storage and retrieval systems. Int. J. Prod. Res. 2013, 50, 7134-7148. [CrossRef]

15. Lerher, T. Travel time model for double-deep shuttle-based storage and retrieval systems. Int. J. Prod. Res. 2015, 54, 2519-2540. [CrossRef]

16. Zou, B.; Xu, X.; Gong, Y.Y.; DeKoster, R. Modeling parallel movement of lifts and vehicles in tier-captive vehicle-based warehousing systems. Eur. J. Oper. Res. 2016, 254, 51-67. [CrossRef]

17. Ning, Z.; Lei, L.; Saipeng, Z.; Lodewijks, G. An efficient simulation model for rack design in multi-elevator shuttle-based storage and retrieval system. Simul. Model. Pract. Theory 2016, 67, 100-116. [CrossRef]

18. Epp, M.; Wiedemann, S.; Furmans, K. A discrete-time queueing network approach to performance evaluation of autonomous vehicle storage and retrieval systems. Int. J. Prod. Res. 2017, 55, 960-978. [CrossRef]

19. Lerher, T. Aisle changing shuttle carriers in autonomous vehicle storage and retrieval systems. Int. J. Prod. Res. 2018, 56, 3859-3879. [CrossRef]

20. Ekren, B.Y.; Akpunar, A.; Sari, Z.; Lerher, T. A tool for time, variance and energy related performance estimations in a shuttle-based storage and retrieval system. Appl. Math. Model. 2018, 63, 109-127. [CrossRef]

21. Ekren, B.Y. A multi-objective optimisation study for the design of an AVS/RS warehouse. Int. J. Prod. Res. 2020. [CrossRef]

22. Ekren, B.Y.; Akpunar, A. An open queuing network-based tool for performance estimations in a shuttle-based storage and retrieval system. Appl. Math. Model. 2021, 89, 1678-1695. [CrossRef]

23. Küçükyaşar, M.; Ekren, B.Y.; Lerher, T. Cost and performance comparison for tier-captive and tier-to-tier SBS/RS warehouse configurations. Int. Trans. Oper. Res. 2021, 28, 1847-1863. [CrossRef]

24. Küçükyaşar, M.; Ekren, B.Y.; Lerher, T. 2021 "Energy Efficient Automated Warehouse Design," Solving Urban Infrastructure Problems Using Smart City Technologies, Handbook on Planning, Design, Development, and Regulation; Vacca, J., Ed.; Morgan Kaufmann: San Francisco, CA, USA, 2020; pp. 269-292, Paperback ISBN 9780128168165; eBook ISBN 9780128168172. 